Check for updates

Cite this: Mater. Adv., 2021, 2, 7369

Received 28th July 2021,

Accepted 28th September 2021

DOI: 10.1039/d1ma00659b

rsc.li/materials-advances

\section{First demonstration of the use of open-shell derivatives as organic luminophores for transparent luminescent solar concentrators $\dagger$}

\author{
Sara Mattiello, (D) a Francesca Corsini, (DD ${ }^{b}$ Sara Mecca, (D) a Mauro Sassi, (D) ${ }^{a}$ \\ Riccardo Ruffo, (D) a Giuseppe Mattioli, (D) ${ }^{c}$ Yohei Hattori, (D) d \\ Tetsuro Kusamoto, (D) *e Gianmarco Griffini (D) ${ }^{\mathrm{b}}$ and Luca Beverina (D)*a
}

\begin{abstract}
Luminescent solar concentrators (LSCs) are rapidly emerging as the key enabling technology towards the realization of zero-energy buildings. The development of new efficient and sustainable luminophores is crucial for further improvement. The large Stokes shift, luminescent and persistent radicals prepared using sustainable routes offer advantages for large-area transparent LSCs featuring minimal colour distortion of the transmitted light. LSCs based on the best performing luminescent radicals are largely unaffected by reabsorption losses, thus providing a better performance with respect to the literature standards over active areas exceeding $400 \mathrm{~cm}^{2}$.
\end{abstract}

\section{Introduction}

Zero-energy buildings combine energy efficiency and on-site renewable energy generation to become independent from external power sources. Solar-cell panels are one of the most established solutions for distributed renewable power generation. Unfortunately, their characteristic aesthetic appearance and their requirement for a specific angle with respect to the incident sunlight limit the available installation area to

\footnotetext{
${ }^{a}$ Department of Materials Science, University of Milano-Bicocca, Via R. Cozzi, 55, Milano, Italy. E-mail: luca.beverina@unimib.it

${ }^{b}$ Department of Chemistry Materials and Chemical Engineering "Giulio Natta", Politecnico di Milano, Piazza Leonardo da Vinci 32, 20133 Milano, Italy. E-mail: gianmarco.griffini@polimi.it

${ }^{c}$ CNR - Istituto di Struttura della Materia, Area della Ricerca di Roma, I-00015 Monterotondo Scalo, Italy

${ }^{d}$ Department of Materials Chemistry, Faculty of Advanced Science and Technology, Ryukoku University, Seta, Otsu, Shiga 520-2194, Japan

${ }^{e}$ Department of Life and Coordination-Complex Molecular Science Institute for Molecular Science 5-1 Higashiyama, Myodaiji, Okazaki, Aichi 444-8787, Japan. E-mail: kusamoto@ims.ac.jp

$\dagger$ Electronic supplementary information (ESI) available: Synthesis and NMR characterization of all new compounds and intermediates. Additional spectroscopic characterization. Computational details. Preparation of LSCs and full optical characterization. See DOI: 10.1039/d1ma00659b
}

rooftops and shelters. Luminescent solar concentrators (LSCs) are regarded as one of the most promising alternative technologies for building integrated photovoltaics, due to their ease of integration. ${ }^{1}$

A planar LSC consists of a high-optical-quality plastic or glass slab that contains or is coated with a luminescent material that is capable of absorbing sunlight and efficiently emitting radiation. Since the refractive index of the slab is higher than that of air, most of the luminescence (depending on the refractive index mismatch between air and host material) is guided by total internal reflection to the edges of the LSC, where it is converted into electricity by efficient solar cells. Fig. 1 shows a schematic representation of the device and the corresponding working principle.

Loss mechanisms belong to two categories: losses related to the matrix material, and processes directly connected with the luminophores. Guiding materials with a high refractive index and good optical quality reduce both the amplitude of the critical angle below which light is reflected instead of being transmitted and the scattering. Poly(methyl methacrylate) (PMMA) is the most common choice, but research on alternative materials is a very active field. ${ }^{2}$ From the standpoint of the luminophore, the critical parameters are the light harvesting efficiency, the luminescence quantum yield, and the separation between the absorption and the emission spectra (the Stokes shift). The Stokes shift is a particularly critical parameter for large-area devices. Even in the case of a near-unity luminescence quantum yield, every absorption and emission event within the slab involves a randomization of the light direction. This causes the loss of part of the emitted light due to the escape cone formed at the slab/air interface. The larger the area, the larger the probability of reabsorption, hence the emphasis on spectral separation over the other chromophore properties, at least for large-area devices. ${ }^{3}$ There are two main classes of LSCs: full-spectrum and transparent devices. Fullspectrum devices are based on luminophores designed to absorb most of the incident solar light. Organic luminophores of the perylene imide family were originally proposed, ${ }^{4-9}$ but they 


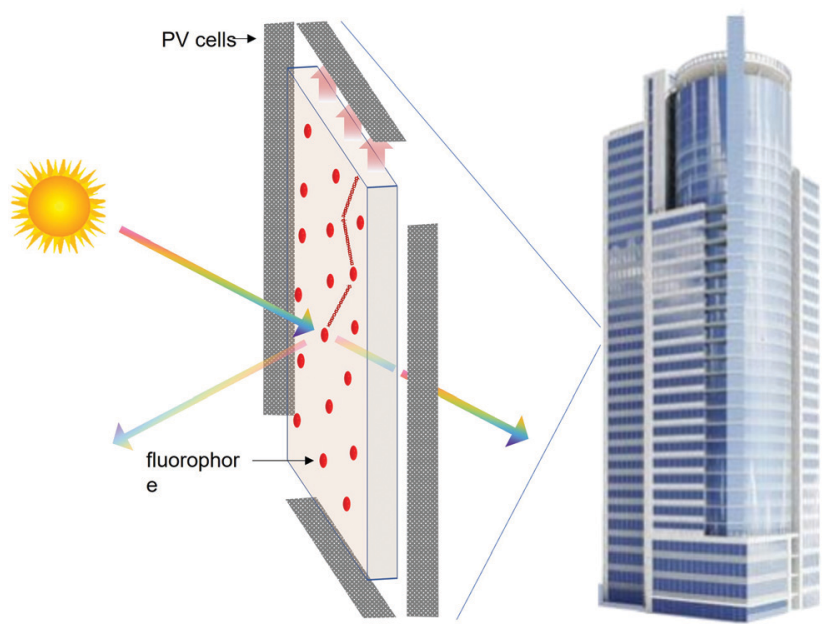

Fig. 1 Working principle of an LSC.

have long been outperformed by semiconducting colloidal quantum dots that offer excellent stability and performance., ${ }^{3,10}$ Schemes based on energy transfer between the emitting species are also possible. ${ }^{11-16}$

Transparent LSC devices are designed to be installed in place of normal windows. They should allow through most of the incident light with only a minor attenuation and, even more importantly, with as little colour distortion as possible. Given such characteristics, transparent LSCs cannot rival fullspectrum devices in terms of their efficiencies. ${ }^{17}$ Suitable luminophores described in the literature feature selective absorption in the UV, NIR, or both spectral regions and belong to the classes of UV-absorbing molecules, ${ }^{18}$ lanthanide chelates ${ }^{19-21}$ iridium complexes, ${ }^{22}$ wide-band-gap colloidal nanocrystals, ${ }^{23,24}$ highly conjugated cyanines, ${ }^{17}$ cycloparaphenylenes (nCPPs), ${ }^{25}$ dual-band UV and NIR absorbers, ${ }^{26}$ non-fullerene acceptors like NIR absorbers, ${ }^{27} \mathrm{UV}$ absorbers featuring aggregation-induced luminescence ${ }^{28,29}$ and recently oxidized [1] benzothieno[3,2-b][1] benzothiophene (BTBT-Ox). ${ }^{30}$

The best luminophores so far described are based on a trade-off between transparency and efficiency and are either light-yellow or blue-coloured due to the presence of residual absorption either at the UV or the NIR edges of the visible spectrum. ${ }^{23,30}$ The alternative solution we describe here is based on the very peculiar spectral features of a class of persistent and luminescent radicals, structurally related to (3,5-dichloro-4-pyridyl)bis(2,4,6-trichlorophenyl)methyl radical (РуBTM). Such new derivatives are characterized by two absorption bands, centred in the UV-vis region.

The UV band is intense, contributes to the power generation and does not impact the visible transparency. The visible-light band is very weak, broad and extends over a sizeable part of the spectrum. As a result, the visible light that is transmitted features the minimal colour distortion characteristics, for example, of sunglasses (Fig. 2).

Such very peculiar spectral characteristics are associated with an exceptionally large Stokes shift, thus making the new

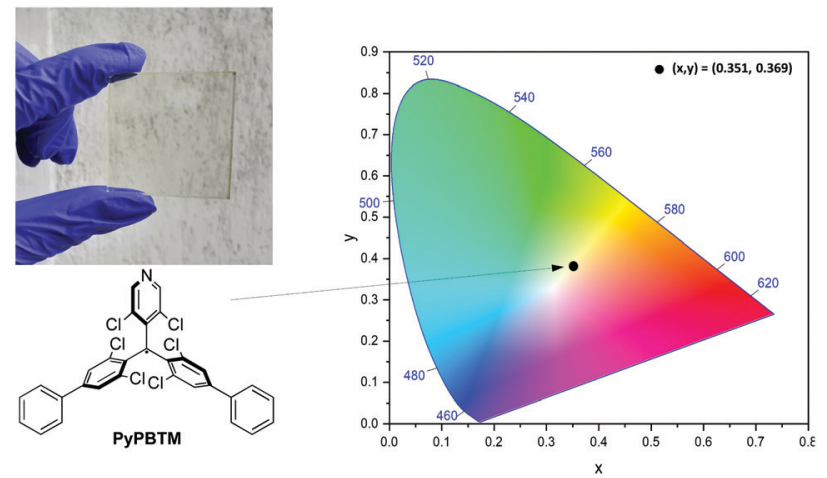

Fig. 2 Colourless LSC obtained using luminescent radical PyPBTM. The chromaticity coordinates of the device $(0.351,0.369)$ are very close to the white point $(0.33,0.33)$, thus ensuring minimal color distortion of the transmitted light.

class of luminophores particularly suitable for the large area required to form transparent, building-integrated smart windows.

\section{Design and synthesis of luminescent radicals}

The newly designed persistent, luminescent radicals are structurally connected with the parent molecule (3,5-dichloro-4pyridyl)bis(2,4,6-trichlorophenyl)methyl radical (PyBTM), a luminescent persistent radical that possesses a quantum yield of $26 \%$ at room temperature in a poly(methyl methacrylate) (PMMA) matrix. The emission efficiency reaches $81 \%$ in diethyl ether: isopentane : ethanol 5:5:2 v/v (i.e., EPA, a mixture of solvents that easily forms glasses upon solidification and thus enables low-temperature optical measurements) at $77 \mathrm{~K} \cdot{ }^{31-35} \mathrm{As}$ mentioned in the introduction, PyBTM features a weak and broad visible absorption, connected with a very large spectral separation with respect to the characteristic red emission $\left(\lambda_{\max }^{\mathrm{Em}}=584 \mathrm{~nm}\right)$. With the aim of improving the lightharvesting capabilities and the overall photostability of PyBTM,${ }^{36}$ we developed three conjugation-extended derivatives that feature two additional aromatic moieties in the $p$-position with respect to the radical centre (Scheme 1).

The different substituents - phenyl (PyPBTM), 6-methoxy-2naphthyl (PyNBTM) and 9-phenanthryl (PyPhBTM) - were selected to lower the optical gap of PуBTM and thus improve the overall light harvesting, while at the same time maintaining a suitable spectral separation. An enhanced conjugation was also expected to positively impact on the overall stability. ${ }^{37}$

All new derivatives can be prepared thanks to a divergent synthesis method that uses PyBTM-H as the common precursor and the Suzuki-Miyaura (S-M) reaction as the preferred arylation protocol. With the aim of connecting sustainability and performance, we based our approach on micellar catalysis. Micellar catalysis enables the substitution of organic solvents with a water solution of suitable surfactants as the reaction medium, and has a very positive effect on the reduction of organic wastes. Once optimized, such a strategy is not only more sustainable but sizeably more efficient as reactions are faster and cleaner, requiring a reduced catalyst loading and 

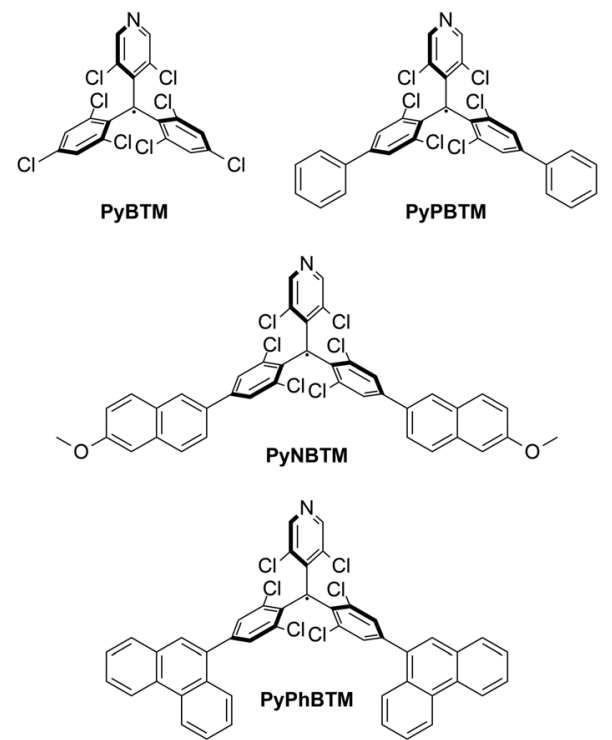

Scheme 1 Chemical structures of PyBTM and the corresponding conjugation-extended derivatives developed as luminophores for LSCs.

mild heating. ${ }^{38-42}$ Some of us have already demonstrated the validity of the approach in the preparation of polyconjugated materials. $^{43-45}$

PyBTM-H is a particularly challenging substrate for S-M coupling in terms of reactivity and selectivity. Chlorine generally requires relatively harsh reaction conditions (high temperature, expensive and/or custom-made phosphines) and, aside from steric hindrance, all 8 chlorine atoms of the molecule are, at least in principle, reactive. Attempts carried out using an organic solvent homogenous solution failed. Selecting the correct surfactant and conditions for a micellar reaction is not trivial, although useful trends are reported in the literature.

The popular designer surfactant TPGS-750M has already demonstrated good performances in promoting the S-M coupling of aryl chlorides. ${ }^{46}$ The industrial surfactant Kolliphor EL (K-EL) proved to be particularly useful in running the reactions under air. ${ }^{43,47}$ The industrial surfactant Tween 80 , when used as a mixture with lecithin (Tween 80/lecithin $8: 2 \mathrm{w} / \mathrm{w}$ - TL82), is helpful whenever reagents/products do not easily disperse in the micellar media. ${ }^{48}$ Scheme $\mathrm{S} 1$ of the ESI $\dagger$ shows the chemical structures of all such surfactants. Table 1 summarizes the results we obtained under a variety of experimental conditions.

Generally, micellar catalysis works best when reagents are hydrophobic. Under such conditions, the selective localization of the reagents in the hydrophobic pocket of the association colloids formed by the surfactants leads to a high local concentration. Reactions become faster and require lower activation temperatures. The case of PyBTM derivatives is unusual and required specific optimization as both the boronic acid and the triarylmethane precursor are somewhat soluble in water at the high $\mathrm{pH}$ that is typical of S-M couplings. Working at r.t., K-EL and TPGS-750M $2 \mathrm{wt} \%$ solutions gave modest and comparable results (40 and 35\% yield, respectively). As previously observed,
Table 1 S-M coupling of PyBTM-H and phenylboronic acid as the function of the reaction medium. Unless otherwise specified, reactions were performed under a nitrogen atmosphere, at $0.3 \mathrm{M} \mathrm{PyBTM-H}$ with 3 eq. of phenylboronic acid, 6 eq. of $\mathrm{N}(E \mathrm{Et})_{3}$ and using $0.08 \mathrm{~mol} \% \mathrm{Pd}$ catalyst

\begin{tabular}{|c|c|c|c|c|}
\hline Entry & Medium & Catalyst & $T\left({ }^{\circ} \mathrm{C}\right)$ & Yield (\%) \\
\hline 1 & $\mathrm{THF} / \mathrm{H}_{2} \mathrm{O} 2: 1 \mathrm{v} / \mathrm{v}$ & $\mathrm{Pd}\left(\mathrm{PPh}_{3}\right)_{4}$ & Reflux & - \\
\hline 2 & K-EL 2 wt $\%$ aq. & $\mathrm{P}(\mathrm{dppf}) \mathrm{Cl}_{2}$ & r.t. & 5 \\
\hline 3 & K-EL 2 wt $\%$ aq. & $\operatorname{Pd}($ amphos $) \mathrm{Cl}_{2}$ & r.t. & 30 \\
\hline 4 & K-EL 2 wt $\%$ aq. & $\mathrm{Pd}(\mathrm{dtbpf}) \mathrm{Cl}_{2}$ & r.t. & 40 \\
\hline $5^{a}$ & K-EL 2 wt $\%$ aq. & $\mathrm{Pd}(\mathrm{dtbpf}) \mathrm{Cl}_{2}$ & r.t. & 40 \\
\hline 6 & TPGS-750M 2 wt $\%$ aq. & $\operatorname{Pd}(\mathrm{dtbpf}) \mathrm{Cl}_{2}$ & r.t. & 35 \\
\hline 7 & TL82 2 wt\% aq. & $\operatorname{Pd}(\mathrm{dtbpf}) \mathrm{Cl}_{2}$ & r.t. & 5 \\
\hline 8 & K-EL 2 wt $\%$ aq. & $\mathrm{Pd}(\mathrm{dtbpf}) \mathrm{Cl}_{2}$ & 70 & 54 \\
\hline 9 & $\mathrm{H}_{2} \mathrm{O}$ & $\mathrm{Pd}(\mathrm{dtbpf}) \mathrm{Cl}_{2}$ & 70 & 50 \\
\hline 10 & K-EL $2 \mathrm{wt} \% /$ toluene $9: 1$ & $\operatorname{Pd}(\mathrm{dtbpf}) \mathrm{Cl}_{2}$ & 70 & 63 \\
\hline
\end{tabular}

K-EL gave the additional advantage of enabling the reactions to be carried out under air (Table 1, entry 5). ${ }^{43}$ Raising the temperature to $70{ }^{\circ} \mathrm{C}$ improved the yield in $\mathrm{K}-\mathrm{EL}$ to $54 \%$ (Table 1 , entry 8 ). Interestingly, plain water (Table 1 , entry 9) also gave a remarkable $50 \%$ yield. This result is in line with the slight solubility of the reagents in water. In the case of troublesome reactions, the literature suggests the introduction of a small amount of an organic cosolvent. ${ }^{49}$ Indeed, the use of a $2 \mathrm{wt} \%$ solution of K-EL in water in the presence of a $10 \mathrm{vol} \%$ of toluene gave by far the best results (entry 10). The use of toluene is a bit of a setback in terms of sustainability, but the amount employed is smaller than that of the combined reagents. The protocol is general and, working under similar conditions, we isolated the triarylmethane precursors PyPBTM-H, PyNBTM-H and PyPhBTM-H in 63, 50 and 35\% yields, respectively.

Conversion to the corresponding luminescent radicals can be performed analogously to the literature protocol for the preparation of PyBTM: deprotonation with ${ }^{t} \mathrm{BuOK}$ in $\mathrm{THF}$, followed by oxidation of the thus-formed carbanion with excess iodine. Filtration using a plug of silica affords PуPBTM, PyNBTM and PyPhBTM in 89, 85 and 80\% yield, respectively (Scheme 2). The formation of the radical species is confirmed by the corresponding EPR spectra, as reported in the ESI. $\dagger$ The behaviour of the three new derivatives is consistent and analogous to that previously reported for the parent PyВTM molecule. ${ }^{33}$

\section{Spectroscopic and computational investigation}

Fig. 3a shows the absorption (continuous line) and normalized emission (dashed line, excitation wavelength $400 \mathrm{~nm}$ ) spectra of all new compounds in chloroform. The ESI $\uparrow$ section reports similar spectra in cyclohexane (Fig. S1). The main spectral features are in line with those previously observed for the PyBTM parent compound and are not significantly solventdependent. The absorption spectra show two bands: one relatively intense and localized in the UV-Vis region, and the other that is weaker and broader and extends over most of the visible spectrum. In all the cases, absorption and emission spectra overlap only marginally due to the relatively low extinction 


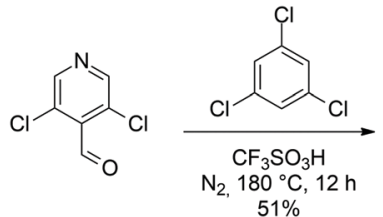

$\mathrm{Ar}=$ Phenyl $(\mathrm{P}) \quad \mid \mathrm{Pd}(\mathrm{dtbpf}) \mathrm{Cl}_{2}, \mathrm{~N}(\mathrm{Et})_{3}$ $\mathrm{N}_{2}, 70^{\circ} \mathrm{C}, 24 \mathrm{~h}$
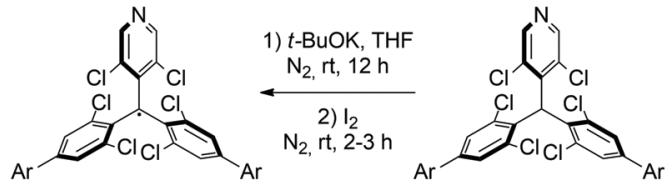

PyPBTM $(89 \%)$ PyNBTM $(85 \%)$

PyPhBTM (80\%)

PyPBTM-H $\quad(63 \%)$

PyNBTM-H $(50 \%)$

PyPhBTM-H $(35 \%)^{a}$ $\mathrm{Ar}=6-\mathrm{MeO}-2-\mathrm{Naphthyl}(\mathrm{N})\} \mathrm{Ar}-\mathrm{B}(\mathrm{OH})_{2} \quad\left(2 \mathrm{wt} \% \mathrm{~K}-\mathrm{EL}\right.$ in $\left.\mathrm{H}_{2} \mathrm{O}\right)$ :toluene $\mathrm{Ar}=9$-Phenanthryl $(\mathrm{Ph}) \quad$ 9:1 $\mathrm{vol} / \mathrm{vol}$

Scheme 2 Synthesis of the luminescent radicals PyPBTM, PyNBTM, and PyPhBTM. ${ }^{a}$ Reaction was performed at $80{ }^{\circ} \mathrm{C}$.

coefficient of the low-energy transition. The extent of the reabsorption losses due to spectral overlap can be quantitatively described according to two different metrics. The radiative overlap (RO) is defined as the fraction of the emitted light that can be reabsorbed by the dye itself. The RO should be as small as possible to ensure minimum reabsorption. ${ }^{50}$ The weighted quality factor $(\langle Q\rangle)$ is given by the ratio between the absorption coefficient at the wavelength of maximum absorbance and the value of the absorption coefficient averaged across the emission region. $\langle Q\rangle$ should be as large as possible to ensure minimum reabsorption. ${ }^{51}$ Both metrics are analytically defined in the ESI $\dagger$ section. Table 2 shows the comparison between the RO and $\langle Q\rangle$ values for PyPBTM and two reference
Table 2 Radiative overlap (RO) and weighted quality factor $(\langle Q\rangle)$ for PyPBTM and the commercially available rylene dyes Lumogen F Violet 570 and Lumogen F Red 305

\begin{tabular}{llr}
\hline Luminophore & RO $(\%)$ & $\langle Q\rangle$ \\
\hline Lumogen F Violet 570 & 9.5 & 20 \\
Lumogen F Red 305 & 8.1 & 6 \\
PyPBTM & 0.8 & 92
\end{tabular}

dyes, Lumogen F Violet 570 and Lumogen F Red 305, which are widely used in full-spectrum and UV-absorbing LSCs, respectively (the chemical structures and the corresponding absorption and emission spectra of the perylene dyes are reported in Fig. S6 of the ESI $\dagger$ ).

The РувтM luminophore shows remarkably better performance (lower spectral overlap) according to both figures of merit. In particular, the RO value of PyBTM is more than 10 times lower than those of the commercial dyes. Also, the extremely high $\langle Q\rangle$ value of the PyBTM luminophore demonstrates that PyBTM is a nearly perfect emitter with negligible contribution of reabsorption losses. Reabsorption phenomena will be discussed further in the section dedicated to testing of the LCS devices.

To gain insight into the origin of the spectral features of the new radicals, we carried out a computational analysis using (time-dependent) density functional theory simulations at the B3LYP level of theory, achieving a good agreement between the experimental and simulated spectra. We included the parent PyBTM species for comparison. The frontier molecular orbitals of all the new derivatives (see Table S1, ESI $\dagger$ ) have a similar origin. Fig. 4 shows the details for PyPhBTM as a representative compound. Unlike the case of PyBTM where the pyridine ring contributes to the population of the HOMO, PyPhBTM features a closed-shell-like HOMO (with practically identical and almost
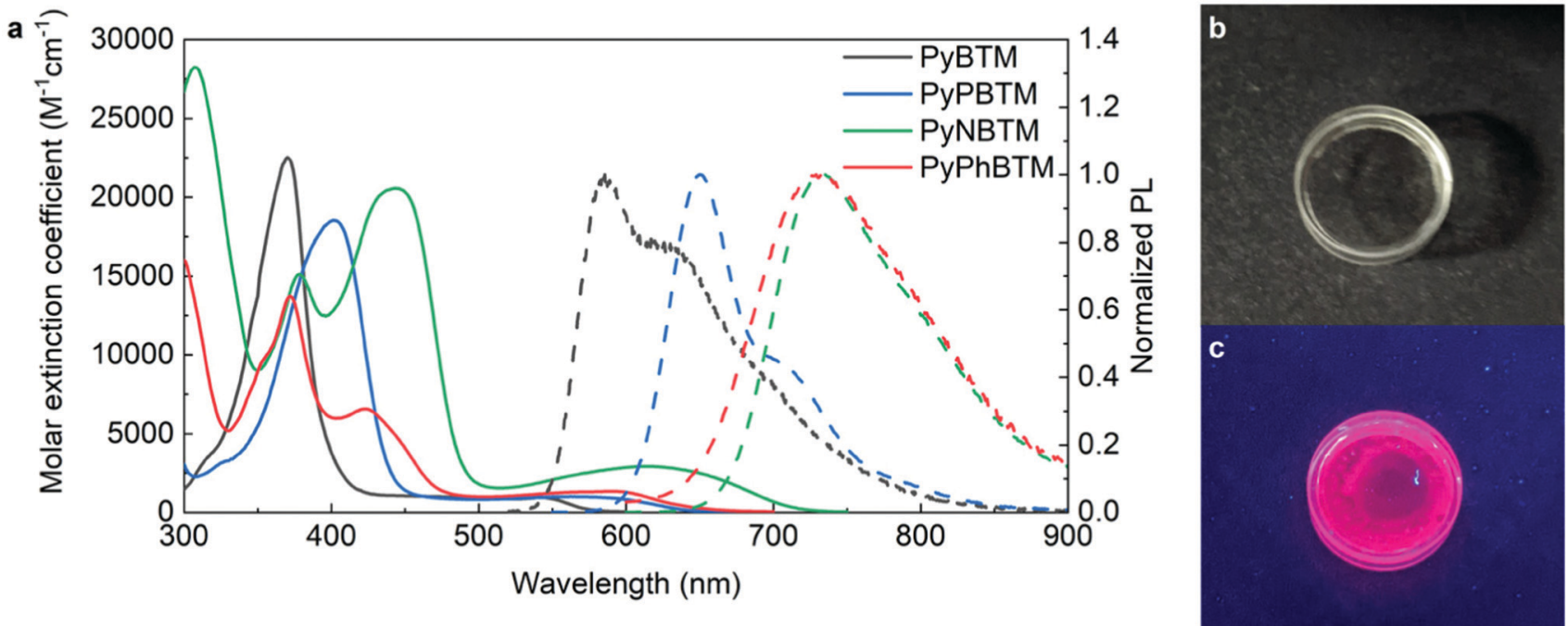

Fig. 3 (a) UV-vis absorption (solid lines) and normalized emission (dashed lines) spectra of luminescent radicals PyBTM, PyPBTM, PyNBTM and PyPhBTM in chloroform. PL spectra were recorded by exciting 0.1 M solutions of PyBTM, PyPBTM, PyNBTM and PyPhBTM at $370 \mathrm{~nm}, 370 \mathrm{~nm}, 445 \mathrm{~nm}$, and $500 \mathrm{~nm}$, respectively. Pictures of a PMMA film containing PyPBTM in a Petri dish under (b) white and (c) UV ( $\lambda=365 \mathrm{~nm})$ light. 

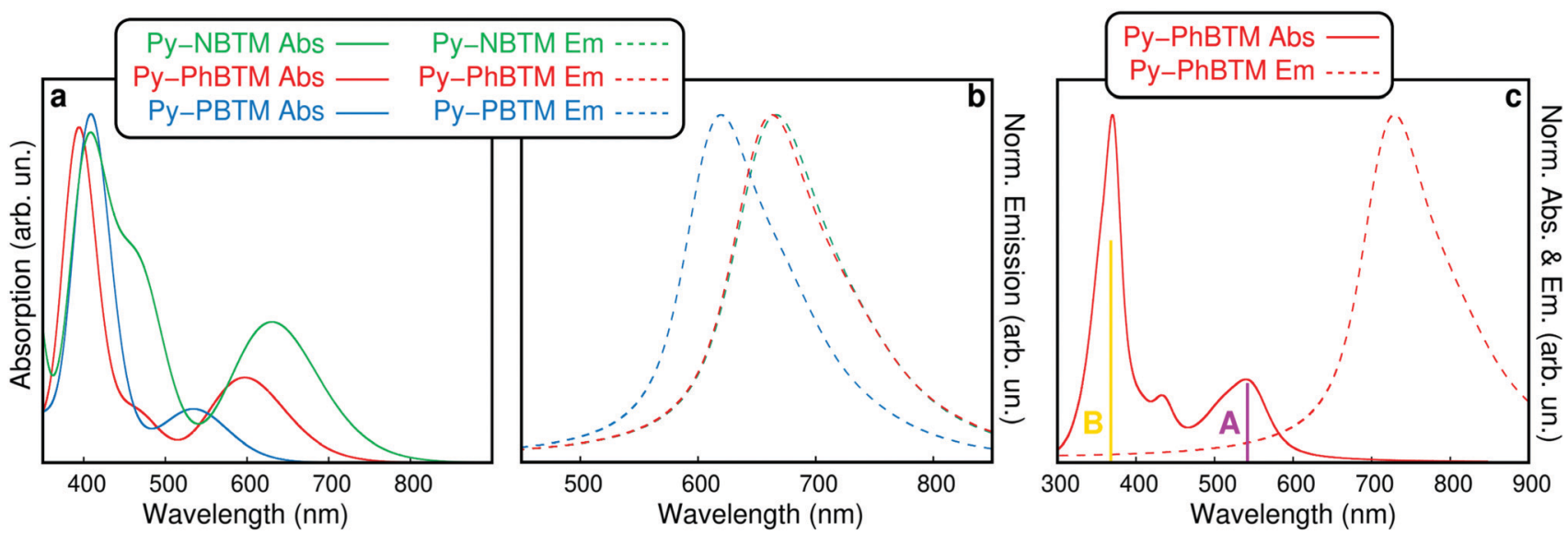

Energy (eV)
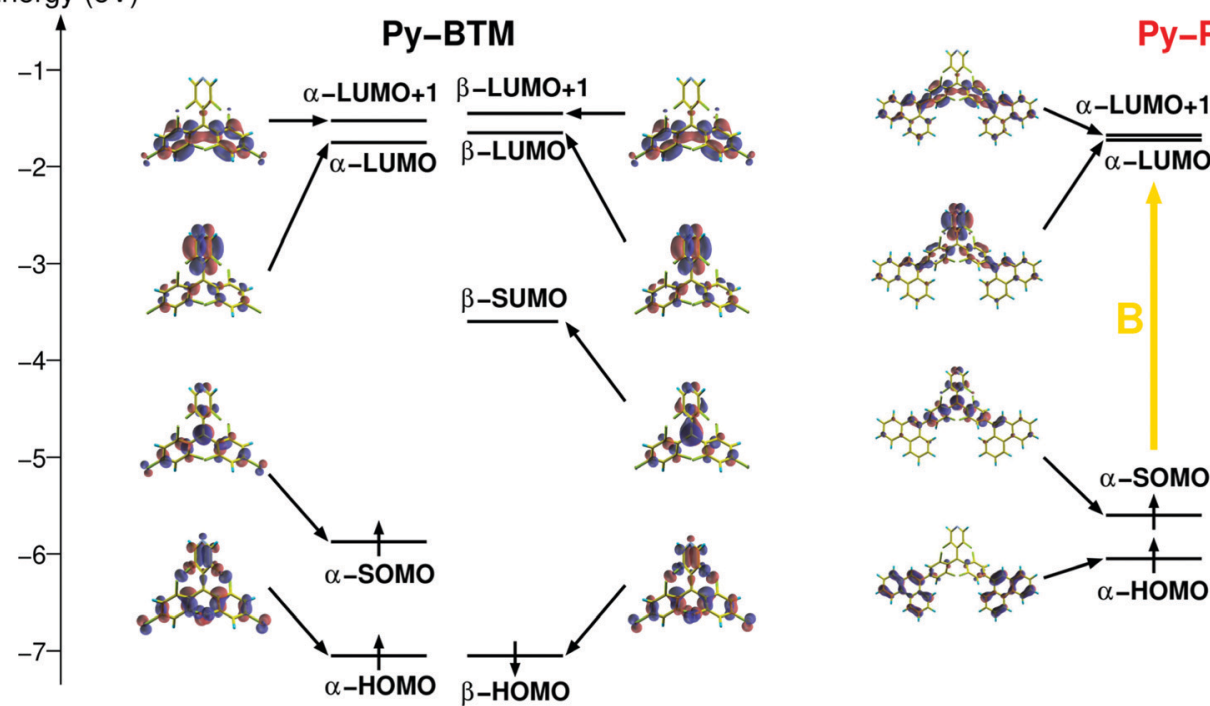

Py-PhBTM

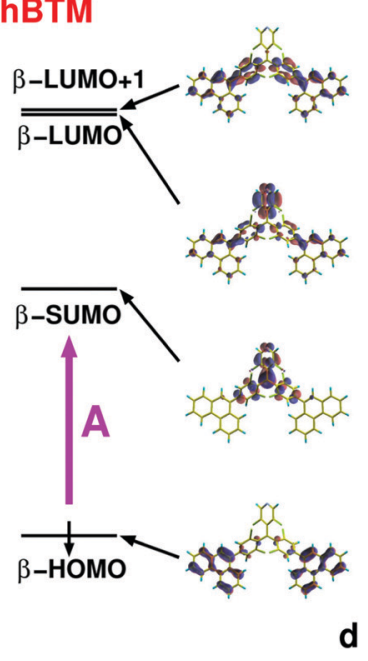

Fig. 4 TDDFT absorption (a) and normalized emission (b) spectra of PyNBTM (green), PyPhBTM (red) and PyPBTM (blue). (c) Normalized TDDFT vibronic absorption and emission spectra of PyPhBTM; a solvatochromic shift has been applied, as discussed in the text. (d) Unrestricted Kohn-Sham molecular orbitals calculated in the case of PyBTM (left) and PyPhBTM (right). The two main transitions in the visible and near-UV regions of the PyPhBTM spectrum ( $A$ and $B$, respectively) have been highlighted in panel (c) and their main contributions in terms of single-particle excitation have been indicated in panel (d).

degenerate $\alpha$ - and $\beta$-HOMOs) localized on the two phenanthrene substituents. The unpaired electron is accommodated in a singly occupied molecular orbital ( $\alpha$-SOMO) within the HOMO-LUMO gap, mainly localized on the radical $\mathrm{C}$ atom, and accompanied by a similar, unoccupied $\beta$-SUMO. A closedshell-like LUMO is mainly localized on the electron-accepting pyridine moiety.

The extension of the conjugation raises in energy the position of the $\alpha$ - and $\beta$-HOMOs compared to PyBTM, but barely affects the position of the other frontier orbitals. Such results are confirmed by the cyclic voltammetry plots reported in the ESI $\dagger$ section. All the new radicals show electrochemical features like those of the parent PyBTM: reversible one-electron oxidation and reduction to the corresponding carbocation and carbanion forms, respectively. The onset of the oxidation wave is shifted towards a less positive potential, in agreement with the donating character of the $p$-substituents along the series
PyNBTM $>$ PyPBTM $>$ PyPhBTM. The reduction waves at negative potentials were essentially identical for the three compounds. Such electronic features are responsible for the optical properties of the molecules. The low energy, weak absorption band (A in Fig. 4c and d) is almost completely (generally around $90 \%$, see Table S1 for details, ESI $\dagger$ ) ascribed to the $\beta$-HOMO $\rightarrow \beta$-SUMO transition involving displacement of the orbital density from the phenylene substituents to the radical. Precisely because of that, the optical gap narrows at an increased effective conjugation length according to the series PyNBTM < PyPhBTM < PyPBTM, as shown in Fig. 4a.

In agreement with the measurements, the TDDFT absorption spectra indicate an increase of the relative intensity of such a transition as the optical gap narrows. This is due to the stronger electron-donor capability of the aryl substituent along the series PyNBTM > PyPhBTM > PyPBTM, yielding excited states with more pronounced charge-separation character. 
On the other hand, the high-energy, strong UV-centred band (B in Fig. 4c and d) is a convolution of different electronic excitations showing an overall prevalent contribution from the $\alpha$-SOMO $\rightarrow \alpha$-LUMO and $\alpha$-SOMO $\rightarrow \alpha$-LUMO+1 transitions (generally around $40-50 \%$, see Table S1 for details, ESI $\dagger$ ). The latter transition is less affected by the introduction of stronger electron-donating groups in the place of chlorine.

As a result, all the transitions in this region are characterized by a moderate red shift with respect to PyBTM, whose strong excitation is in the near-UV peaks at $370 \mathrm{~nm}$ (TDDFT excitation calculated at $376 \mathrm{~nm}$ ). Although they are slightly blue-shifted by around $1200 \mathrm{~cm}^{-1}$, probably because the calculations do not reproduce solvatochromic effects, relative emission spectra shown in Fig. 4b agree nicely with the measurements.

Indeed, as shown in Fig. 4c, if we take into account the vibronic contributions to the electronic transitions and the solvatochromic shift, we obtain a better match between the calculations and measurements. Further details on the optical properties of all the investigated molecules are included in Table S1 (ESI $\dagger$ ).

All the new radicals are luminescent at room temperature. The emission quantum yields in chloroform for PyPBTM $(8.6 \%$, $\left.\tau_{\mathrm{f}}=12.04 \mathrm{~ns}\right)$ and PyNBTM $\left(6.4 \%, \tau_{\mathrm{f}}=3.86 \mathrm{~ns}\right)$ outperform that of the parent PyBTM (2.8\%). PyPhBTM $\left(0.9 \%, \tau_{\mathrm{f}}=0.82 \mathrm{~ns}\right)$ is less efficient. The emission peaks are progressively red-shifted according to the same trend observed for the absorption spectra.

PyBTM possesses a remarkable temperature-dependence in the emission efficiency; as such we studied the temperaturedependence of the emission spectra of all the new radicals using EPA as the solvent (see Fig. S2 of the ESI $\dagger$ ). In all cases, we observed a sizeable improvement in the luminescence intensity associated with a non-monotonous variation of the emission maximum. We consistently observed an emission red shift on lowering the temperature from 298 to $140 \mathrm{~K}$, followed by a blue shift when further lowering the temperature down to $77 \mathrm{~K}$. Such a blue shift was also associated with a more pronounced vibronic structure, which is probably due to vitrification of the solvent. The structural relaxation of the radicals in the photoexcited state becomes more restricted, decreasing the reorganization energy, thus resulting in the blue shift of the emission. The $77 \mathrm{~K}$ emission quantum yield was in all cases sizeably improved, although not as much as previously observed for PувтM. In particular, РуРвTM was the most efficient in the series $\left(\Phi_{\mathrm{f}}=22 \%, \tau_{\mathrm{f}}=21.5 \mathrm{~ns}\right.$ in EPA), followed by PyNBTM $\left(\Phi_{\mathrm{f}}=16 \%, \tau_{\mathrm{f}}=7.8 \mathrm{~ns}\right.$ in EPA $)$ and PyPhBTM $\left(\Phi_{\mathrm{f}}=7 \%, \tau_{\mathrm{f}}=5.6 \mathrm{~ns}\right.$ in EPA). The temperature dependence of the luminescence quantum yield has already been discussed and stems from the rotovibrational thermalization pathways becoming progressively more relevant as the temperature is increased. ${ }^{33}$

At $77 \mathrm{~K}$, when the solvent is vitrified, the molecules are surrounded by a very rigid environment. The very same effect is also responsible for the relatively high luminescence quantum yield of PyBTM in a PMMA solid matrix (26\%).

PMMA is the matrix of choice for the fabrication of luminescent solar concentrators ${ }^{2}$ we thus prepared a solid sample
Table 3 Main spectral characteristics of the luminescent radicals PyBTM, PyPBTM, PyNBTM and PyPhBTM in chloroform and EPA. All the spectra are recorded in $0.1 \mathrm{M}$ solutions. Excitation wavelengths were $370 \mathrm{~nm}$, 445 nm, and 500 nm for PyPBTM, PyNBTM and PyPhBTM, respectively. Measurements on PyBTM and PMMA from ref. 27. Full spectra in EPA and $\mathrm{PL}$ decays for lifetime determination are shown in the ESI

\begin{tabular}{|c|c|c|c|c|c|}
\hline Compound (medium) & $T(\mathrm{~K})$ & $\begin{array}{l}\lambda_{\max }^{\mathrm{Abs}} \\
(\mathrm{nm})\end{array}$ & $\begin{array}{l}\lambda_{\max }^{\mathrm{Em}} \\
(\mathrm{nm})\end{array}$ & $\Phi_{\mathrm{f}}(\%)$ & $\tau_{\mathrm{f}}(\mathrm{ns})$ \\
\hline $\operatorname{PyBTM}^{a}\left(\mathrm{CHCl}_{3}\right)$ & 298 & 541 & 585 & 2.8 & 6.4 \\
\hline $\operatorname{PyBTM}^{a}$ (EPA) & 77 & - & 570 & 81 & - \\
\hline PyBTM $^{a}$ (PMMA) & 298 & - & - & 26 & - \\
\hline PyPBTM $\left(\mathrm{CHCl}_{3}\right)$ & 298 & 567 & 650 & 8.6 & 12.0 \\
\hline PyPBTM (EPA) & 77 & - & 644 & 22 & 21.5 \\
\hline PyPBTM (PMMA) & 298 & - & 645 & 12 & 15.5 \\
\hline PyNBTM $\left(\mathrm{CHCl}_{3}\right)$ & 298 & 618 & 735 & 6.4 & 3.9 \\
\hline PyNBTM (EPA) & 77 & - & 714 & 16 & 7.8 \\
\hline PyPhBTM $\left(\mathrm{CHCl}_{3}\right)$ & 298 & 585 & 729 & 0.9 & 0.8 \\
\hline PyPhBTM (EPA) & 77 & - & 688 & 7 & 5.6 \\
\hline
\end{tabular}

of PyPBTM - the compound offering the highest room-temperature luminescence and the largest spectral separation of the series - in PMMA (Fig. 3b). As is shown in Fig. 3c, we obtained red emission with a room-temperature quantum yield of $12 \%$, and a lifetime of 15.5 ns. Such values are still significantly smaller than those that characterise the state-of-the-art compounds and work needs to be done to further improve them. The literature suggests that the introduction of stronger electron donors on the aryl substituents could improve the luminescence. ${ }^{52}$ Table 3 summarizes all the relevant spectral features of the luminescent radicals.

Aside from a large spectral separation, minimal distortion of the transmitted light and high emission efficiency, luminophores for applications in LSC technology must be thermally and photochemically stable. ${ }^{53}$ This is considered a major shortcoming for radicals; that is, the chemical species are considered rather more as reaction intermediates than final compounds. PyBTM, having a half-life of 4.1 min under continuous irradiation at $370 \mathrm{~nm}$ in air, represented a breakthrough with respect to previous examples of persistent radicals, although it remains critically short with respect to the expectations of the field that is used to the extreme ruggedness of perylene dyes. ${ }^{33}$

As is shown in Fig. 5, all the new conjugation-extended derivatives feature dramatically improved photostability, probably because of the more pronounced delocalization of the radicals over the aryl substituents. The comparative enhancement in the half-life values of the new derivatives measured under comparable conditions were 77 for PyPBTM (half-life $313 \mathrm{~min}$ ), 93 for PyNBTM (half-life $381 \mathrm{~min}$ ) and 1052 for PyPhBTM (4313 min, 72 h), see the ESI $\dagger$ section for full details. Such numbers may still be considered unsuitable for LSC applications, but it should be stressed that PMMA itself is strongly absorbing at $370 \mathrm{~nm}$, thus providing efficient photoprotection. ${ }^{54}$ As we will discuss in the following section, further encapsulation is possible and beneficial.

\section{Transparent colourless LSCs}

Based on the obtained results, we prepared lab-scale thin-film LSC demonstrators using PMMA as the host matrix and 


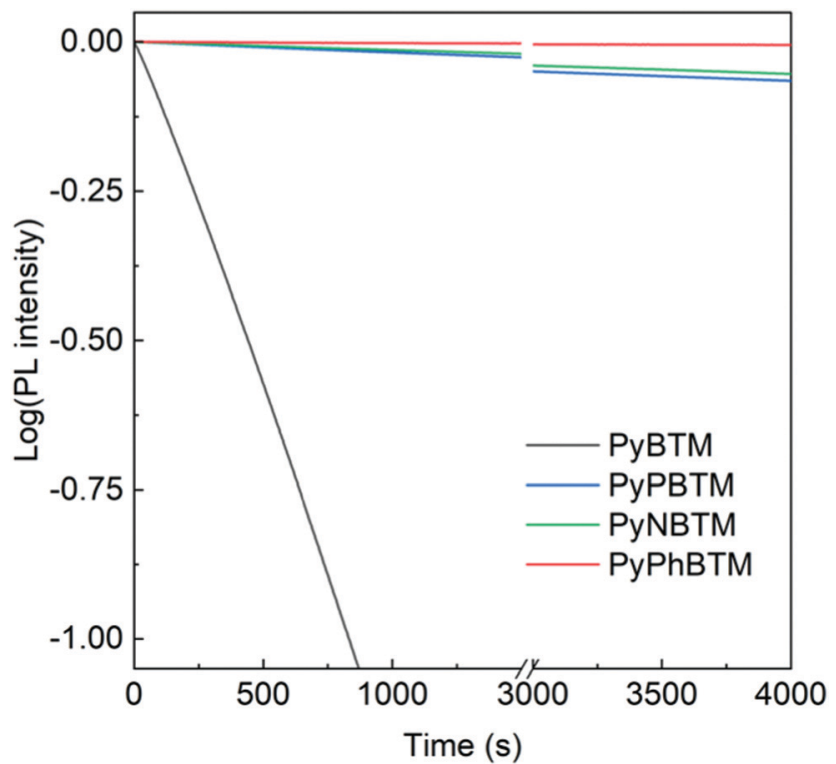

Fig. 5 Comparative stability of luminescent radicals in $\mathrm{CH}_{2} \mathrm{Cl}_{2}$ solution under irradiation at $370 \mathrm{~nm}$.

PyPBTM of varying concentrations as the luminophore. For increased absorbance levels, the intensity of the corresponding emission spectra recorded in the front-face configuration grew steadily up to a maximum that was found at a $3 \mathrm{wt} \%$ loading, after which the luminescence decreased (see Fig. S8 of the ESI $\dagger$ ). These trends suggest the presence of dissipative phenomena in strongly doped LSCs $(>3 \mathrm{wt} \%$, absorbance $>1$ in the 350-500 nm wavelength range) causing a portion of the absorbed photons to be lost via non-radiative pathways. Such losses are connected with two phenomena: the formation of poorly emissive molecular aggregates within the matrix, or reabsorption events caused by the small but not completely negligible overlap between the absorption and emission spectra. ${ }^{55}$ It is widely accepted that the self-absorption dissipative processes are the key loss mechanisms for the LSCs. ${ }^{56}$ To gain further insight into this aspect, we calculated the selfabsorption efficiency $\eta_{\text {self }}$ values from the obtained edgeemission spectra at various luminophore concentrations. In the absence of the self-absorption phenomena, $\eta_{\text {self }}$ will tend to unity. As shown in Fig. $6, \eta_{\text {self }}$ decreased with the increase in maximum absorbance $\left(A_{\max }\right)$ following an exponential decay that could be fitted by $\eta_{\text {self }}=0.55+0.33 \exp \left(-0.50 A_{\max }\right)$.

Accordingly, a maximum $\eta_{\text {self }}=0.88$ can be predicted for a very low absorbance $\left(A_{\max } \rightarrow 0\right)$, where self-absorption is expected to be negligible. On the other hand, in highly concentrated LSCs (high $A_{\max }$ ) self-absorption effects result in a minimum $\eta_{\text {self }}=0.55$ (i.e., $45 \%$ of the emitted photons will be lost due to self-absorption). Even the value obtained at high $A_{\max }$ is remarkably better than the best ones $\left(\eta_{\text {self }}=0.2-0.4\right)$ characterizing the established luminophores used in fullspectrum devices like the perylene derivative Lumogen F Red $305 .^{57,58}$ This behaviour indicate the potential of these novel luminescent persistent radicals as a new class of luminophores

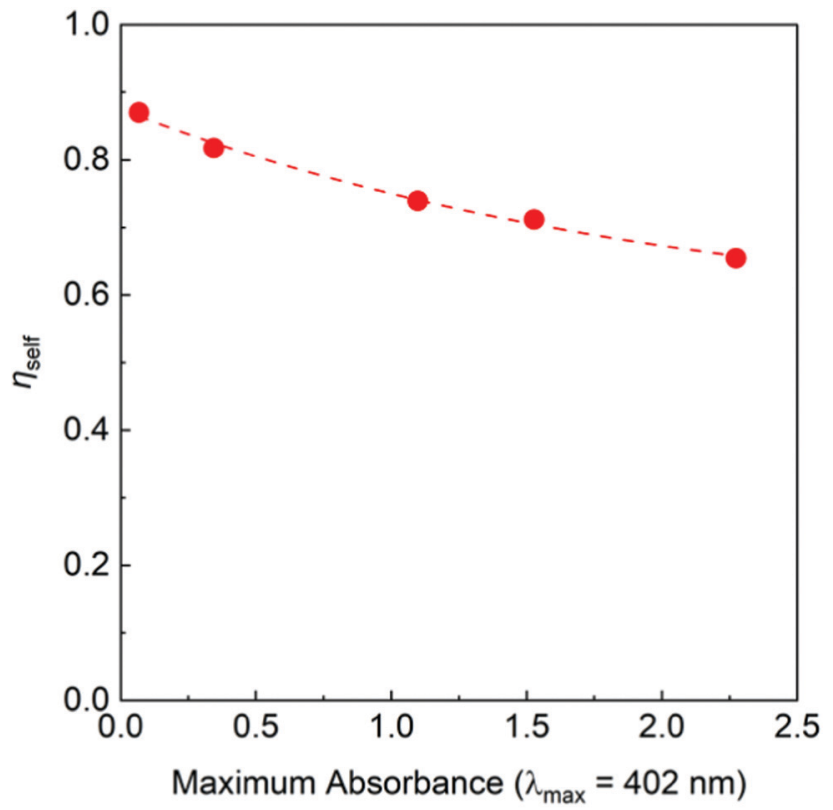

Fig. 6 Self-absorption efficiency of the thin-film PyPBTM/PMMA LSCs as a function of maximum absorbance (viz. luminophore concentration). The dashed lines correspond to the exponential fit to the experimental data with the function $\eta_{\text {self }}=0.55+0.33 \exp \left(-0.50 A_{\text {max }}\right)$.

for large-area devices, where reabsorption events can considerably impact the output performance. We assessed the optical response of PyPBTM/PMMA LSC devices as light-harvesting and -conversion photonic systems using two key figures of merit: the external photon efficiency $\eta_{\text {ext }}$, defined as the ratio of the total output photon flux measured at the four edges of the LSC with respect to the incident photon flux, and the internal photon efficiency $\eta_{\text {int }}$, given by the total output photon flux measured at the four edges of the LSC relative to the absorbed photon flux (definitions and calculations are reported in the ESI $†)^{59}$

As shown in Fig. 7, $\eta_{\text {ext }}$ increased with the PyPBTM concentration, reaching a maximum $\eta_{\mathrm{ext}} \approx 0.50 \%$ at $3 \mathrm{wt} \%$. At higher loadings, $\eta_{\text {ext }}$ decreased slightly, in line with the optical loss pathways occurring at high doping levels, as described above. On the other hand, a maximum $\eta_{\text {int }}=5.14 \%$ was observed for highly diluted systems (PyPBTM $\approx 0.1 \mathrm{wt} \%$ ), followed by a slight decrease to values in the range of $3.3-3.6 \%$ for luminophore concentrations of 1-3 wt $\%$. For higher loadings, a progressive decrease in $\eta_{\text {int }}$ was detected, which is probably attributable to increasingly relevant quenching losses (nonradiative relaxation pathways) occurring within the system under these conditions.

The limited but non-negligible overlap between the absorption and emission spectra of PyPBTM in the 600-650 nm wavelength range suggests that reabsorption processes may play a role in decreasing the optical performance of the LSCs. In addition, scattering losses within the host matrix during photon transport and light guiding can also affect the overall device performance. ${ }^{60,61}$ To investigate this phenomenon more in detail, we evaluated the light-transport efficiency 


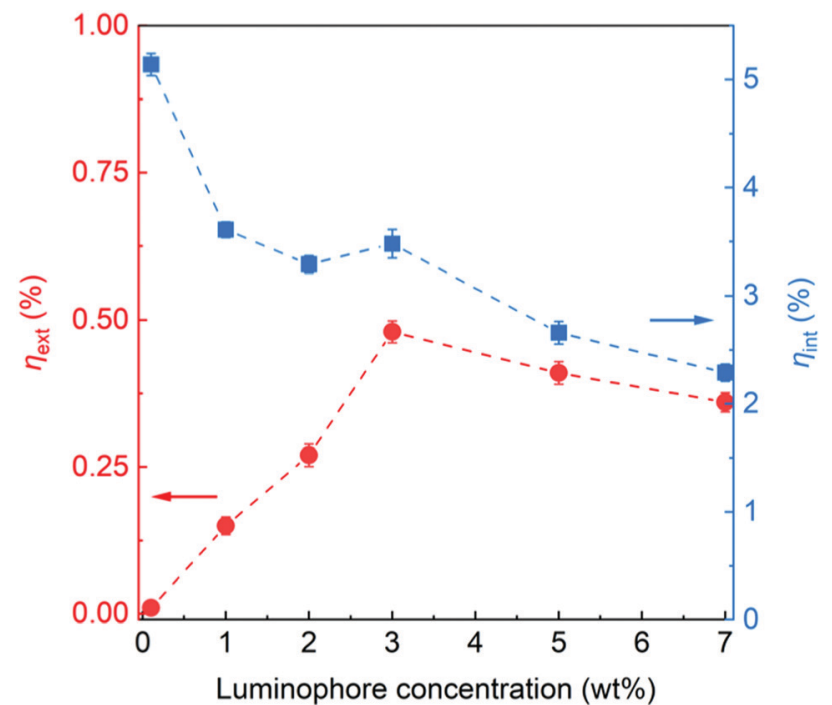

Fig. 7 External photon efficiency $\eta_{\text {ext }}$ and internal photon efficiency $\eta_{\text {int }}$ vs. luminophore concentration.

in PyPBTM/PMMA LSCs at the optimal luminophore concentration (3wt\%, Fig. 7) by recording their single-edge optical output power upon illumination at increasingly larger distances from the collecting edge (details in the ESI $\dagger$ ). As shown in Fig. 8 , a $\sim 10 \%$ loss of performance was experimentally recorded at optical distances of up to $20 \mathrm{~cm}$ from the illumination spot, clearly suggesting the negligible contribution of luminophore reabsorption losses and scattering on the output performance. To further extend the analysis, we also conducted

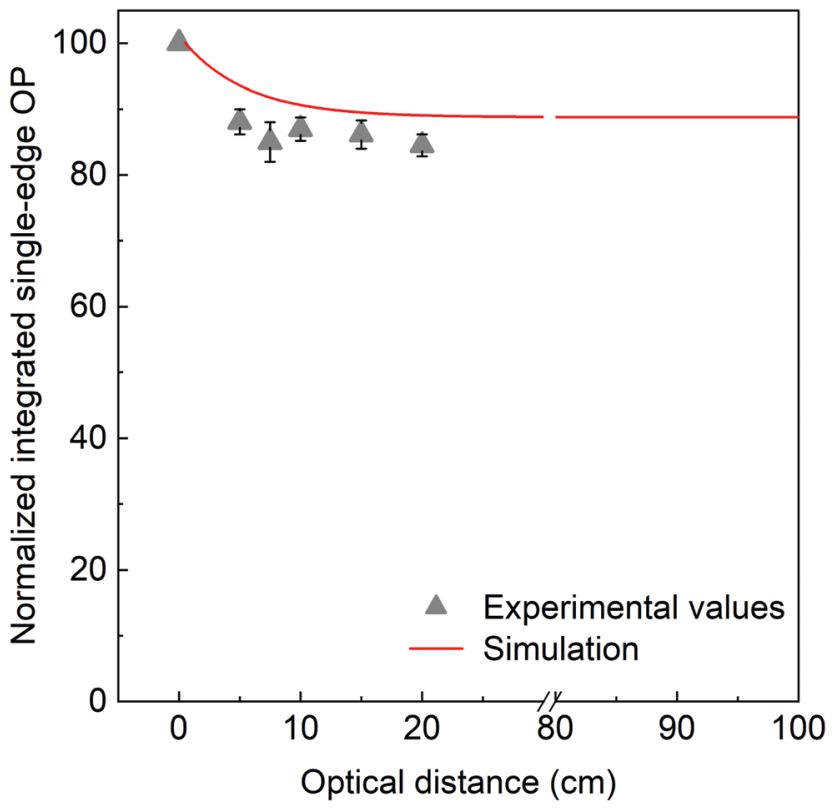

Fig. 8 Normalized intensity decay of the integrated optical power output as a function of optical distance from the illumination spot under 1 Sun top irradiation: experimental values (triangles) and simulated values (solid line). Monte Carlo ray-tracing simulation has been carried out on an ideal LSC that is free from scattering and reabsorption losses.
Monte Carlo ray-tracing simulations on the same LSCs of up to $1 \mathrm{~m}$ lateral size. In addition to the excellent match with the experimental results for small $(<20 \mathrm{~cm})$ devices, the simulation showed that a constant output performance could be achieved, even on the up-scaled systems. This confirms the potential of this new class of luminophores for large-area reabsorptionfree LSC devices, notwithstanding the relatively limited PyPBTM/PMMA LSC-PV assembly device efficiency under 1 Sun full illumination, mainly associated with the spectral response of the luminescent radical species (see ESI $\dagger$ ).

Finally, in view of their prospective outdoor use, we also undertook a preliminary assessment of the long-term photostability of PyPBTM/PMMA-based LSCs by monitoring the optical performance of the devices over time under UV-A light illumination (see ESI $\dagger$ for details).

Despite the highly aggressive weathering conditions (highenergy photons, and exposure in a non-inert atmosphere), encouraging trends were observed for such systems, especially in the presence of designer host matrices previously shown to hamper photoinduced degradation and prevent luminophore emission quenching. ${ }^{62,63}$

Combining recent findings in high-durability host-matrix materials $^{2,8,64}$ and advanced encapsulation approaches for $\mathrm{PVs}^{65-67}$ is expected to further boost the outdoor photostability of these organic luminescent radical systems, ultimately proving their application potential in the LSC field.

\section{Conclusions}

We have developed three new conjugation-enhanced luminescent radicals, structurally derived from the parent PyBTM, a persistent radical already widely employed as both a single molecule and building block in a variety of advanced applications. We designed the new radicals to profit from the two most favourable features of PуBTM - its efficient luminescence and the high spectral separation between its absorption and emission spectra - while further boosting their light harvesting in the visible region and their photostability. The overall features of the newly synthesized materials are particularly suitable for applications in transparent LSCs for integration into buildings.

From the standpoint of synthesis, our approach is both efficient, since we employed a divergent scheme using РуBTM as the common precursor for the new materials, and sustainable, as we fully exploited the advantages of micellar catalysis in reducing the amount of organic solvents required.

In terms of properties, we succeeded in sizeably extending the visible-light absorption of the derivatives, while at the same time increasing by orders of magnitude their stability to continuous UV light exposure. While embedded in thin film LSCs, PyPBTM - the most efficient derivative of the series - demonstrated a particularly good performance in terms of reduction of reabsorption losses. In fact, while the lateral dimensions of the best-performing LSCs based on standard perylene dyes are limited to $10-20 \mathrm{~cm}$ due to strong reabsorption, the PyPBTM-based devices show only a minor size-dependent 
performance ( $\sim 10 \%$ loss) with near constant output response, even on up-scaled (up to $20 \mathrm{~cm}$ ) systems. This evidence clearly suggests the negligible contribution of luminophore reabsorption losses and scattering on the output performance. To further highlight the applicability of such a technology to building integrated photovoltaics, the colour coordinates of the PyPBTM devices are close to the white point so as to ensure negligible colour distortion of the transmitted light.

\section{Author contributions}

Sara Mattiello, Sara Mecca and Mauro Sassi: synthesis of all compounds, and optimization of the micellar catalysis methods. Francesca Corsini: preparation and characterization of the LSC devices. Giuseppe Mattioli: computational studies. Riccardo Ruffo: electrochemical characterization. Yohei Hattori: solution characterization of the luminescent radicals, including low temperature data, and EPR measurements. Gianmarco Griffini, Tetsuro Kusamoto and Luca Beverina: conceptualization, discussion of the data, drafting of the manuscript and overall coordination.

\section{Conflicts of interest}

There are no conflicts to declare.

\section{Notes and references}

1 F. Meinardi, F. Bruni and S. Brovelli, Nat. Rev. Mater., 2017, 2, 17072 .

2 G. Griffini, Front. Mater., 2019, 6, 1-8.

3 F. Meinardi, A. Colombo, K. A. Velizhanin, R. Simonutti, M. Lorenzon, L. Beverina, R. Viswanatha, V. I. Klimov and S. Brovelli, Nat. Photonics, 2014, 8, 392-399.

4 R. Turrisi, A. Sanguineti, M. Sassi, B. Savoie, A. Takai, G. Patriarca, M. Salamone, R. Ruffo, G. Vaccaro, F. Meinardi, T. Marks, A. Facchetti and L. Beverina, J. Mater. Chem. A, 2015, 3, 8045-8054.

5 L. Beverina and A. Sanguineti, Sol. Cell Nanotechnol., 2013, 317-355.

6 A. Sanguineti, M. Sassi, R. Turrisi, R. Ruffo, G. Vaccaro, F. Meinardi and L. Beverina, Chem. Commun., 2013, 49, 1618-1620.

7 W. E. Benjamin, D. R. Veit, M. J. Perkins, E. Bain, K. Scharnhorst, S. McDowall, D. L. Patrick and J. D. Gilbertson, Chem. Mater., 2014, 26, 1291-1293.

8 D. Pintossi, A. Colombo, M. Levi, C. Dragonetti, S. Turri and G. Griffini, J. Mater. Chem. A, 2017, 5, 9067-9075.

9 F. Corsini, E. Tatsi, A. Colombo, C. Dragonetti, C. Botta, S. Turri and G. Griffini, Nano Energy, 2021, 80, 105551.

10 F. Meinardi, H. McDaniel, F. Carulli, A. Colombo, K. A. Velizhanin, N. S. Makarov, R. Simonutti, V. I. Klimov and S. Brovelli, Nat. Nanotechnol., 2015, 10, 878-885.

$11 \mathrm{~J}$. ter Schiphorst, A. M. Kendhale, M. G. Debije, C. Menelaou, L. M. Herz and A. P. H. J. Schenning, Chem. Mater., 2014, 26, 3876-3878.
12 S. Flores Daorta, A. Proto, R. Fusco, L. Claudio Andreani and M. Liscidini, Appl. Phys. Lett., 2014, 104, 153901.

13 D. Cambié, F. Zhao, V. Hessel, M. G. Debije and T. Noël, Angew. Chem., 2017, 129, 1070-1074.

14 J. L. Banal, K. P. Ghiggino and W. W. H. Wong, Phys. Chem. Chem. Phys., 2014, 16, 25358-25363.

15 O. Altan Bozdemir, S. Erbas-Cakmak, O. O. Ekiz, A. Dana and E. U. Akkaya, Angew. Chem., Int. Ed., 2011, 50, 10907-10912.

16 G. Fortunato, E. Tatsi, F. Corsini, S. Turri and G. Griffini, ACS Appl. Polym. Mater., 2020, 2, 3828-3839.

17 C. Yang and R. R. Lunt, Adv. Opt. Mater., 2017, 5, 1600851. 18 J. Xu, B. Zhang, M. Jansen, L. Goerigk, W. W. H. Wong and C. Ritchie, Angew. Chem., Int. Ed., 2017, 56, 13882-13886.

19 X. Wang, T. Wang, X. Tian, L. Wang, W. Wu, Y. Luo and Q. Zhang, Sol. Energy, 2011, 85, 2179-2184.

20 A. Sanguineti, A. Monguzzi, G. Vaccaro, F. Meinardi, E. Ronchi, M. Moret, U. Cosentino, G. Moro, R. Simonutti, M. Mauri, R. Tubino and L. Beverina, Phys. Chem. Chem. Phys., 2012, 14, 6452-6455.

21 S. F. H. Correia, V. de Zea Bermudez, S. J. L. Ribeiro, P. S. André, R. A. S. Ferreira and L. D. Carlos, J. Mater. Chem. A, 2014, 2, 5580-5596.

22 V. Fiorini, N. Monti, G. Vigarani, G. Santi, F. Fasulo, M. Massi, L. Giorgini, A. B. Muñoz-García, M. Pavone, A. Pucci and S. Stagni, Dyes Pigm., 2021, 193, 109532.

23 F. Meinardi, Q. A. Akkerman, F. Bruni, S. Park, M. Mauri, Z. Dang, L. Manna and S. Brovelli, ACS Energy Lett., 2017, 2, 2368-2377.

24 C. S. Erickson, L. R. Bradshaw, S. McDowall, J. D. Gilbertson, D. R. Gamelin and D. L. Patrick, ACS Nano, 2014, 8, 3461-3467.

25 P. Della Sala, N. Buccheri, A. Sanzone, M. Sassi, P. Neri, C. Talotta, A. Rocco, V. Pinchetti, L. Beverina, S. Brovelli and C. Gaeta, Chem. Commun., 2019, 55, 3160-3163.

26 C. Yang, W. Sheng, M. Moemeni, M. Bates, C. K. Herrera, B. Borhan and R. R. Lunt, Adv. Energy Mater., 2021, 11, 2003581.

27 C. Yang, M. Moemeni, M. Bates, W. Sheng, B. Borhan and R. R. Lunt, Adv. Opt. Mater., 2020, 8, 1901536.

28 J. L. Banal, J. M. White, T. W. Lam, A. W. Blakers, K. P. Ghiggino and W. W. H. Wong, Adv. Energy Mater., 2015, 5, 1500818.

29 J. L. Banal, J. M. White, K. P. Ghiggino and W. W. H. Wong, Sci. Rep., 2014, 4, 4635.

30 S. Mattiello, A. Sanzone, F. Bruni, M. Gandini, V. Pinchetti, A. Monguzzi, I. Facchinetti, R. Ruffo, F. Meinardi, G. Mattioli, M. Sassi, S. Brovelli and L. Beverina, Joule, 2020, 4, 1988-2003.

31 B. Carlotti, A. Cesaretti, P. L. Gentili, A. Marrocchi, F. Elisei and A. Spalletti, Phys. Chem. Chem. Phys., 2016, 18, 23389-23399.

32 S. Kimura, M. Uejima, W. Ota, T. Sato, S. Kusaka, R. Matsuda, H. Nishihara and T. Kusamoto, J. Am. Chem. Soc., 2021, 143, 4329-4338.

33 Y. Hattori, T. Kusamoto and H. Nishihara, Angew. Chem., Int. Ed., 2014, 53, 11845-11848. 
34 S. Kimura, T. Kusamoto, S. Kimura, K. Kato, Y. Teki and H. Nishihara, Angew. Chem., Int. Ed., 2018, 57, 12711-12715.

35 K. Kato, S. Kimura, T. Kusamoto, H. Nishihara and Y. Teki, Angew. Chem., Int. Ed., 2019, 58, 2606-2611.

36 M. O. Reese, S. A. Gevorgyan, M. Jørgensen, E. Bundgaard, S. R. Kurtz, D. S. Ginley, D. C. Olson, M. T. Lloyd, P. Morvillo, E. A. Katz, A. Elschner, O. Haillant, T. R. Currier, V. Shrotriya, M. Hermenau, M. Riede, K. R. Kirov, G. Trimmel, T. Rath, O. Inganäs, F. Zhang, M. Andersson, K. Tvingstedt, M. Lira-Cantu, D. Laird, C. McGuiness, S. J. Gowrisanker, M. Pannone, M. Xiao, J. Hauch, R. Steim, D. M. Delongchamp, R. Rösch, H. Hoppe, N. Espinosa, A. Urbina, G. Yaman-Uzunoglu, J.-B. Bonekamp, A. J. J. M. van Breemen, C. Girotto, E. Voroshazi and F. C. Krebs, Special Issue: 3rd International Summit on OPV Stability, 2011, vol. 95, pp. 1253-1267.

37 R. G. Hicks, Org. Biomol. Chem., 2007, 5, 1321-1338.

38 S. Handa, Y. Wang, F. Gallou and B. H. Lipshutz, Science, 2015, 349, 1087-1091.

39 S. Handa, E. D. Slack and B. H. Lipshutz, Angew. Chem., Int. Ed., 2015, 54, 11994-11998.

40 B. H. Lipshutz and A. R. Abela, Org. Lett., 2008, 10, 5329-5332.

41 S. Handa, M. P. Andersson, F. Gallou, J. Reilly and B. H. Lipshutz, Angew. Chem., Int. Ed., 2016, 55, 4914-4918.

42 S. Handa, J. D. Smith, M. S. Hageman, M. Gonzalez and B. H. Lipshutz, ACS Catal., 2016, 6, 8179-8183.

43 S. Mattiello, M. Rooney, A. Sanzone, P. Brazzo, M. Sassi and L. Beverina, Org. Lett., 2017, 19, 654-657.

44 A. Sanzone, A. Calascibetta, M. Monti, S. Mattiello, M. Sassi, F. Corsini, G. Griffini, M. Sommer and L. Beverina, ACS Macro Lett., 2020, 9, 1167-1171.

45 A. Sanzone, A. Calascibetta, E. Ghiglietti, C. Ceriani, G. Mattioli, S. Mattiello, M. Sassi and L. Beverina, J. Org. Chem., 2018, 83, 15029-15042.

46 B. S. Takale, R. R. Thakore, N. M. Irvine, A. D. Schuitman, X. Li and B. H. Lipshutz, Org. Lett., 2020, 22, 4823-4827.

47 M. Rooney, S. Mattiello, R. Stara, A. Sanzone, P. Brazzo, M. Sassi and L. Beverina, Dyes Pigm., 2018, 149, 893-901.

48 C. Ceriani, E. Ghiglietti, M. Sassi, S. Mattiello and L. Beverina, Org. Process Res. Dev., 2020, 24(11), 2604-2610.
49 C. M. Gabriel, N. R. Lee, F. Bigorne, P. Klumphu, M. Parmentier, F. Gallou and B. H. Lipshutz, Org. Lett., 2016, 19, 194-197.

50 D. Alonso-Álvarez, D. Ross, E. Klampaftis, K. R. McIntosh, S. Jia, P. Storiz, T. Stolz and B. S. Richards, Prog. Photovoltaics Res. Appl., 2015, 23, 479-497.

51 V. I. Klimov, T. A. Baker, J. Lim, K. A. Velizhanin and H. McDaniel, ACS Photonics, 2016, 3, 1138-1148.

52 A. Abdurahman, T. J. H. Hele, Q. Gu, J. Zhang, Q. Peng, M. Zhang, R. H. Friend, F. Li and E. W. Evans, Nat. Mater., 2020, 19, 1224-1229.

53 G. Griffini, L. Brambilla, M. Levi, M. Del Zoppo and S. Turri, Sol. Energy Mater. Sol. Cells, 2013, 111, 41-48.

54 K. G. de Castro. Monsores, A. O. da Silva, S. de Sant'ana. Oliveira, J. G. P. Rodrigues and R. P. Weber, J. Mater. Res. Technol., 2019, 8, 3713-3718.

55 G. Griffini, M. Levi and S. Turri, Renewable Energy, 2015, 78, 288-294.

56 J. Roncali, Adv. Energy Mater., 2020, 10, 2001907.

57 T. Dienel, C. Bauer, I. Dolamic and D. Brühwiler, Sol. Energy, 2010, 84, 1366-1369.

58 E. Tatsi, G. Fortunato, B. Rigatelli, G. Lyu, S. Turri, R. C. Evans and G. Griffini, ACS Appl. Energy Mater., 2020, 3, 1152-1160.

59 M. G. Debije, R. C. Evans and G. Griffini, Energy Environ. Sci., 2021, 14, 293-301.

60 F. Corsini, A. Nitti, E. Tatsi, G. Mattioli, C. Botta, D. Pasini and G. Griffini, Adv. Opt. Mater., 2021, 9, 2100182.

61 R. D. Breukers, G. J. Smith, H. L. Stirrat, A. J. Swanson, T. A. Smith, K. P. Ghiggino, S. G. Raymond, N. M. Winch, D. J. Clarke and A. J. Kay, Appl. Opt., 2017, 56, 2630-2635.

62 G. Griffini, M. Levi and S. Turri, Prog. Org. Coat., 2014, 77, 528-536.

63 G. Griffini, M. Levi and S. Turri, Sol. Energy Mater. Sol. Cells, 2013, 118, 36-42.

64 G. Griffini and S. Turri, J. Appl. Polym. Sci., 2016, 133, 43080.

65 J.-M. Delgado-Sanchez, Sol. Energy Mater. Sol. Cells, 2019, 202, 110134.

66 J. Ahmad, K. Bazaka, L. J. Anderson, R. D. White and M. V. Jacob, Renewable Sustainable Energy Rev., 2013, 27, 104-117.

67 F. Corsini and G. Griffini, J. Phys. Energy, 2020, 2, 031002. 